\title{
Inventory simulation tools: Separating nuclide contributions to radiological quantities
}

\author{
Mark R. Gilbert ${ }^{\mathrm{a}}$, Michael Fleming, and Jean-Christophe Sublet \\ United Kingdom Atomic Energy Authority, Culham Science Centre, Abingdon, Oxfordshire OX14 3DB, UK
}

\begin{abstract}
The activation response of a material is a primary factor considered when evaluating its suitability for a nuclear application. Various radiological quantities, such as total (becquerel) activity, decay heat, and $\gamma$ dose, can be readily predicted via inventory simulations, which numerically evolve in time the composition of a material under exposure to neutron irradiation. However, the resulting data sets can be very complex, often necessarily resulting in an over-simplification of the results - most commonly by just considering total response metrics. A number of different techniques for disseminating more completely the vast amount of data output from, in particular, the FISPACT-II inventory code system, including importance diagrams, nuclide maps, and primary knock-on atom (PKA) spectra, have been developed and used in scoping studies to produce database reports for the periodic table of elements. This paper introduces the latest addition to this arsenal standardised and automated plotting of the time evolution in a radiological quantity for a given material separated by contributions from dominant radionuclides. Examples for relevant materials under predicted fusion reactor conditions, and for bench-marking studies against decay-heat measurements, demonstrate the usefulness and power of these radionuclide-separated activation plots.
\end{abstract}

\section{Introduction}

The selection of materials for nuclear applications, such as planned fusion research reactors, future fusion power plants, or advanced GEN-IV fission systems, is a complex process with many factors to consider. Not only must a material withstand extreme mechanical and thermal conditions, but it must do this while being bombarded by high-energy neutrons that displace the constituent atoms and alter the properties of the material. Furthermore, some of the nuclear interactions cause the very nature of the atoms to change, via transmutation, with potential additional impacts on original performance. Finally, and perhaps most importantly, these transmutations must not produce too many highly-active radioactive nuclides, because reactors must be manned by personnel during maintenance operations and the amount of radioactivewaste disposal required is a strong factor in both the political and commercial feasibility of nuclear reactors.

Whilst these activation, transmutation and damage responses can be calculated accurately for a given material in a detailed reactor design, there is often an additional need, at the material selection stage, to understand the general response of pure materials. Scoping calculations, across all pure elements, have been performed at UKAEA using the FISPACT-II [1] inventory simulation platform, combined with the latest libraries of nuclear cross section and decay data, for both fusion and fission neutron irradiation environments. An automated simulation infrastructure has been developed, not only to run the scoping simulations with FISPACT-II, but also to post-process the complex results into informative

a e-mail: mark.gilbert@ukaea.uk visualization formats, such as importance diagrams [2,3], nuclide maps [3], or plots of primary knock-on atom (PKA) spectra [4]. Even the collation of the various output presentations for a set of materials into a single report can be automated, avoiding the need to manually enter potentially thousands of images in a document. All of this has been achieved using open-source, command-linedriven software, including GNUPLOT [5] for plotting and ${ }_{\mathrm{LT}} \mathrm{T} \mathrm{X}$ for document typesetting. The automated infrastructure allows calculations to be readily repeated for multiple irradiation scenarios, and, more importantly, provides the platform to take advantage of the latest, advanced nuclear data libraries, which are more complete than their predecessors and are constantly being updated.

Several scoping studies have been performed, the newest of which is a materials handbook for the naturally occurring elements up to $\mathrm{Bi}$ under predicted plasmafacing first wall (FW) fusion DEMOnstration power plant conditions, simulated with the latest TENDL2015 neutron-induced reaction library [6] (another new handbook, considering the response of pure elements and selected alloys and compounds under ITER conditions, has also recently been released [7]). That handbook [6], required almost 60,000 separate inventory simulations, is nearly 800 pages long, and contains more than 1500 separate figures and several hundred tables. Full details of the calculations and various output formats are given in the report itself. Rather than repeating those details here, this paper instead focuses on a new feature for the 2016 fusion handbook [6]: plots that give separate, individual radionuclide contributions to the radiological response (total becquerel activity, decay heat, or contact $\gamma$ dose rate) of interest. Examples for relevant materials under predicted DEMO fusion reactor conditions, and for 


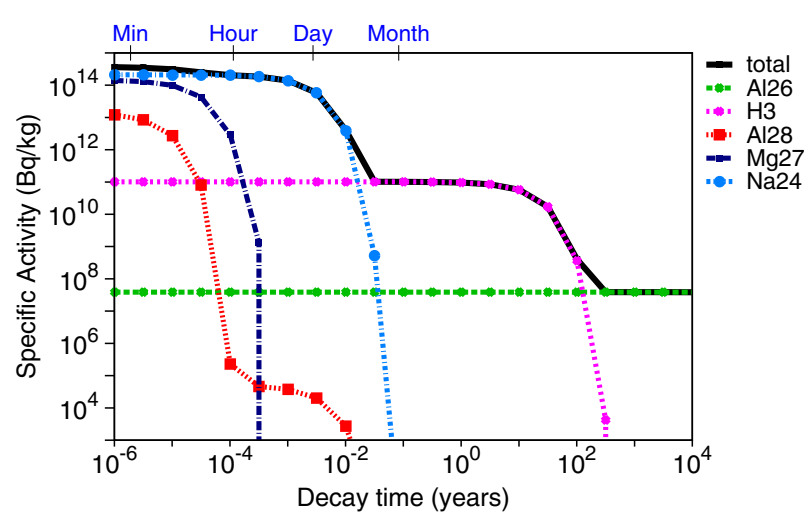

Figure 1. Radioisotope-separated aluminium activity response during cooling following a 2-year irradiation in a predicted first-wall DEMO fusion reactor environment.

bench-marking studies against decay-heat measurements, are also discussed below, demonstrating the power and potential application of such presentations.

\section{Radionuclide-separated decay plots}

Understanding the breakdown of radionuclides contributing to activity as a function of decay time is an important step in the analysis of activated materials, especially when comparing to experiment, where, for example, gamma spectroscopy may allow separation of activity from different nuclides. Furthermore, it can give a prediction, for a material or alloy of interest, that a particular radionuclide causes unwanted activity at some stage during decaycooling following irradiation. The analysis may then also provide a potential solution as to how this unwanted activity could be avoided - for example, by the removal or reduction of the constituent element or isotope whose presence led to the production of the problem nuclide.

For the above reasons automatic generation of graphical representations of the radionuclide-separated, time-evolving activity of a material following irradiation have been prototyped for inclusion into the FISPACT-II [1] inventory system.

Figures 1, 2, and 3, show examples of such plots for pure $\mathrm{Al}, \mathrm{Mo}$, and $\mathrm{Ti}$, respectively, after two-year simulated irradiations with FISPACT-II and TENDL-2015 [6] in a typical FW DEMOnstration fusion power plant spectrum, where the usual $14 \mathrm{MeV}$ fusion neutron peak was the main contributor to a total flux of $5 \times 10^{14} \mathrm{n} \mathrm{cm}^{-2} \mathrm{~s}^{-1}$ (see [15] for more details). In each graph the time evolution in total becquerel activity is plotted as a solid black curve, alongside similarly time-evolving curves for the important individual radionuclides that contribute to the activity of the material at any stage during its decaycooling (FISPACT-II automatically computes the list of nuclides that should be included). Note that any of the other radiological quantities output by FISPACT-II, such as decay heat or contact $\gamma$ dose rate, can also be separated by nuclide and plotted in this way.

Plots like these have been produced before (see for example [8]), but it would have most likely required significant effort to extract and plot the relevant data from the raw inventory output, where there may be several hundred lines of text for each time-step (each time-step provides a data point for every curve in the

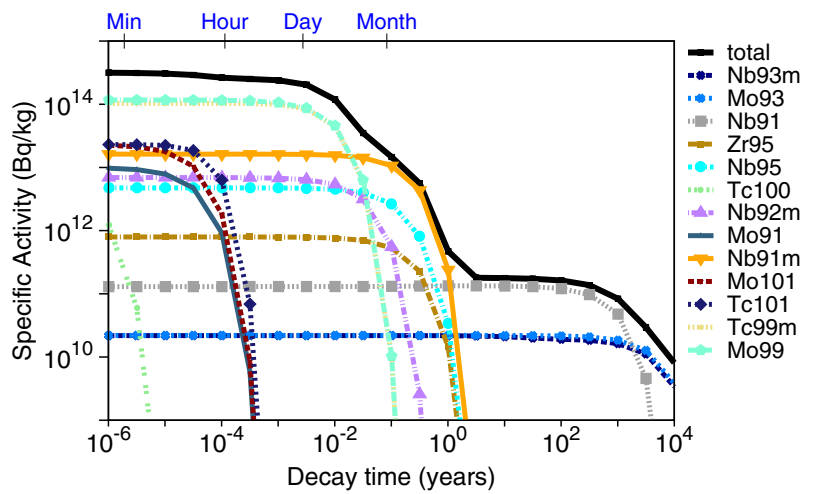

Figure 2. Radioisotope-separated molybdenum activity response during cooling following a 2-year irradiation in a predicted first-wall DEMO fusion reactor environment.

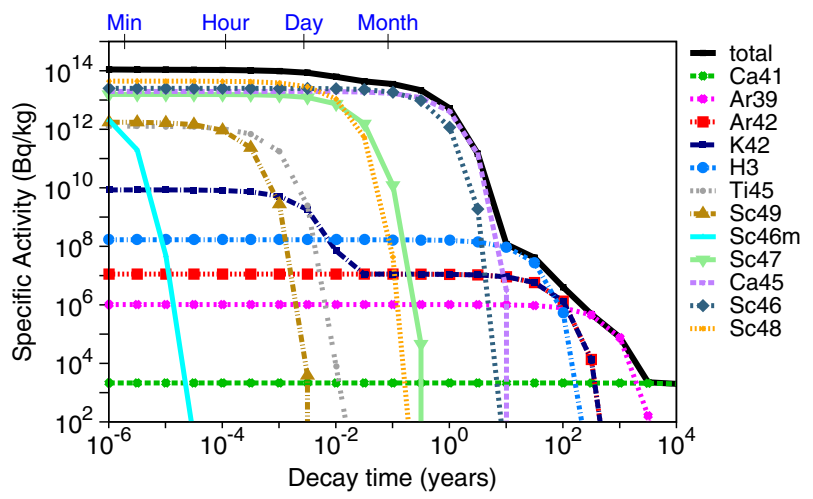

Figure 3. Radioisotope-separated titanium activity response during cooling following a 2-year irradiation in a predicted firstwall DEMO fusion reactor environment.

figures). This is not practical for regular use, particularly for large scoping studies such as those required for the aforementioned handbooks [6], and so it was vital to develop the new routines to automatically generate the required data formats and (GNUPLOT [5]) plot scripts for these plots. This automation is now fully incorporated into the FISPACT-II code system as a standard feature (via a new code word NUCGRAPH - see [1] for details) - thereby allowing for the usual command-line taskscripting -, making graphs of this kind a 'default' output to immediately present the important features of a material's radiological response.

For example, Fig. 1 is straightforwardly interpretable showing that at decay times of less than a few days ${ }^{24} \mathrm{Na}$ (half-life $T_{1 / 2}=15$ hours) is the dominant radionuclide in $\mathrm{Al}$, producing the majority of activity. At longer decay times, up to around 100 years, tritium ${ }^{3} \mathrm{H}\left(T_{1 / 2}=\right.$ 12.3 years) is dominant, which could be particularly problematic if aluminium were to be used in a near-plasma fusion environment where tritium is already present in large amounts. At very long cooling times, ${ }^{26} \mathrm{Al}\left(T_{1 / 2}=\right.$ $7 \times 10^{5}$ years) becomes the only contributor to activity in irradiated $\mathrm{Al}$.

Meanwhile, in Fig. 2, for Mo, which is a potential alternative high-heat flux material to the ubiquitously studied tungsten [3], two radionuclides contribute almost equally to the activity over decay-times of less than around five days. This is despite the fact that one of the nuclides, ${ }^{99 m} \mathrm{Tc}$, only has a half-life of 6 hours, but is completely 

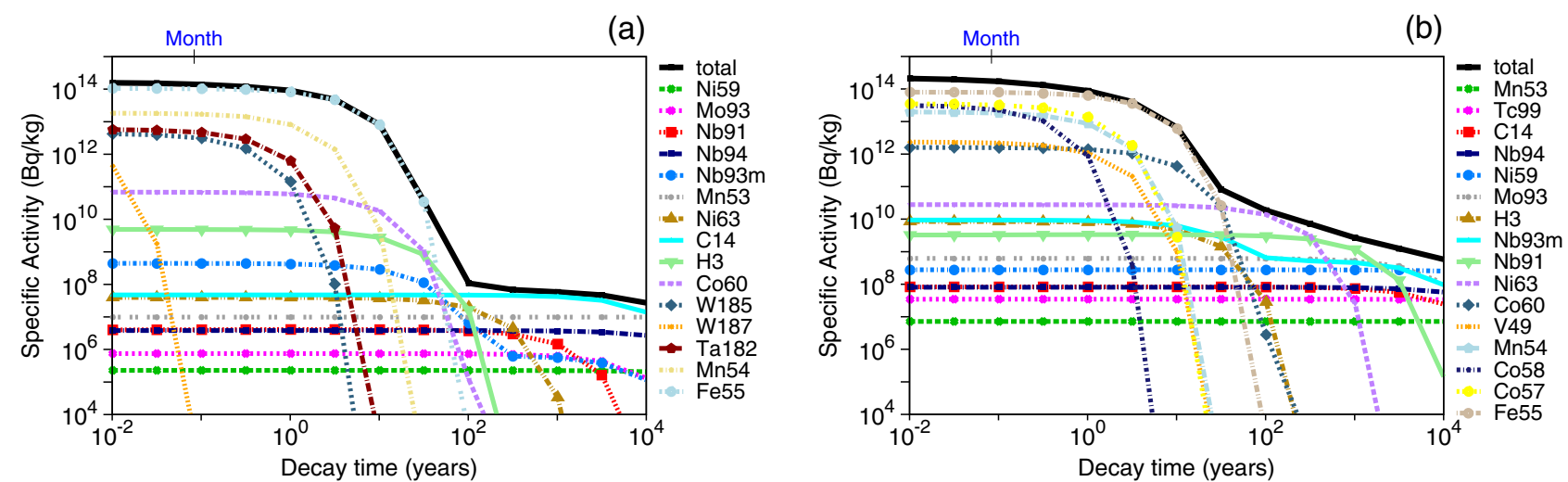

Figure 4. Radioisotope-separated activity response during cooling following a 2-year irradiation in a predicted first-wall DEMO fusion reactor environment of (a) Eurofer steel, and (b) SS316L(N) (ITER grade) [11].

understandable when one realises that the other nuclide ${ }^{99} \mathrm{Mo}$, with a much longer, 2.75 day half-life, $\beta^{-}$-decays to the former. At longer decay times, first the metastable ${ }^{91 m} \mathrm{Nb}\left(T_{1 / 2}=61\right.$ days $)$ and then the ground state ${ }^{91} \mathrm{Nb}$ ( $T_{1 / 2}=680$ years) are the main radionuclides producing activity in DEMO-irradiated Mo.

Even in more complicated examples, such as that for $\mathrm{Ti}$ in Fig. 3, where there are seemingly many nuclides contributing significantly to the activity in a complicated plot, it is nonetheless possible to discern the important features. In Fig. 3, one can see that ${ }^{48} \mathrm{Sc}\left(T_{1 / 2}=1.8\right.$ days $)$ is the primary radionuclide during the first day of cooling, followed by ${ }^{46} \mathrm{Sc}\left(T_{1 / 2}=84\right.$ days $)$ and ${ }^{45} \mathrm{Ca}\left(T_{1 / 2}=\right.$ 163 days), with the latter, in particular, important up to decay times of around one year. There then follows a cooling period of ${ }^{3} \mathrm{H}$ dominance, followed by a period of ${ }^{39} \operatorname{Ar}\left(T_{1 / 2}=269\right.$ years $)$, and then lastly, at very long decay times, ${ }^{41} \mathrm{Ca}\left(T_{1 / 2}=1 \times 10^{5}\right.$ years $)$.

For this last $\mathrm{Ti}$ case, at short decay times in particular, this kind of immediate understanding would not have been possible in other visualization methodologies due to overlapping contributions from several nuclides, which demonstrates the usefulness of such plots - provided, as developed here, they are easy to generate.

\subsection{Alloy examples}

Materials for real-world applications are not normally the pure elemental examples considered above. In practice a material will often be a complex alloy comprised of many different elements. Steels in particular, are usually finely-tuned compositions containing as many as 20 or more elements. In such situations it becomes even more important to be able to understand, visually or otherwise, which particular nuclides will be problematic in nuclear environments, and hence which elements of the composition might need to be altered.

For example, Fig. 4, shows the typical activity response of two of the primary steels envisaged for nuclear fusion power plants - the well-known SS316, and Eurofer, which is designed to be "low-activation" $[9,10]$. As before, both steels, whose compositions were taken from the baseline specifications for the under-construction ITER experimental fusion device (see [11]), were irradiated for 2 years in the DEMO FW spectrum and then their activation response during cooling was charted. The figures immediately show that Eurofer (Fig. 4a) performs better (has a lower activity) at long cooling times, particularly for 100-year decay times and beyond, which demonstrates that it would produce significantly less long-term waste than SS316. However, the plots provide extra information - showing that the high activity in SS316 is predominantly caused by two isotopes of nickel, ${ }^{63} \mathrm{Ni}\left(T_{1 / 2}=101\right.$ years $)$ and ${ }^{59} \mathrm{Ni}\left(T_{1 / 2}=7.6 \times\right.$ $10^{4}$ years). These are insignificant in Eurofer due to its very low Ni content ( $0.01 \mathrm{wt} . \%$ compared to around $12 \%$ in SS316). An isotope of niobium also contributes, ${ }^{91} \mathrm{Nb}$ ( $T_{1 / 2}=680$ years), created mainly by transmutation of Mo (see Fig. 2), which is 2.5 wt.\% of the input SS316 considered here, but only $0.003 \%$ of Eurofer.

\subsection{Decay-heat bench-marking}

The radionuclide breakdown can also be useful when bench-marking the results from inventory simulations against measured experiments. For example, a series of decay-heat experiments were performed at the Fusion Neutron Source (FNS) facility at JAEA [12]. These experiments have been simulated using FISPACTII and various international nuclear data libraries. The resulting (simulated) decay-heat cooling has been validated against the decay-heat measurements recorded during the experiment (see for example [13]).

In Fig. 5a, the experimental data points obtained after a five minute irradiation of pure $\mathrm{Nb}$ are compared to the simulated results using TENDL-2015 and another wellknown library, ENDF/B-VII.1 [14]. Clearly, ENDF/BVII.1 has failed to capture the experiment, and the radionuclide contribution to decay-heat plot for the TENDL-2015 simulation in Fig. 5b reveals the reason why. It shows that there are a number of isomeric states, ${ }^{90 m} \mathrm{Y},{ }^{94 m} \mathrm{Nb}$, and ${ }^{92 m} \mathrm{Nb}$, that dominate the decay heat during the 1-hour experimental timescale, which are absent from the equivalent plot (not shown) for ENDF/B-VII.1. Indeed, with ENDF/B-VII.1, only the ${ }^{90} \mathrm{Y}$ is present in the simulation, explaining the decay-heat level shown in Fig. 5a for that library.

\section{Summary}

The breakdown of isotopic contributions to the radiological responses of materials following neutron irradiation is a vital part of the analyses performed during material selection-studies and waste-production 
(a)

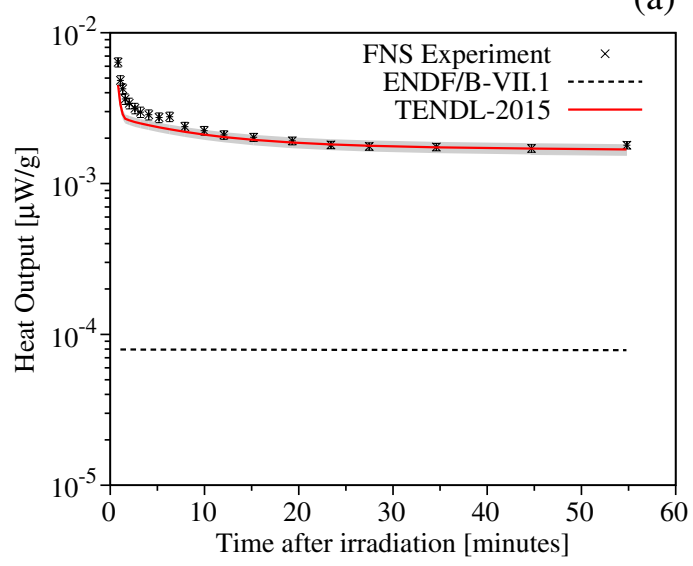

(b)

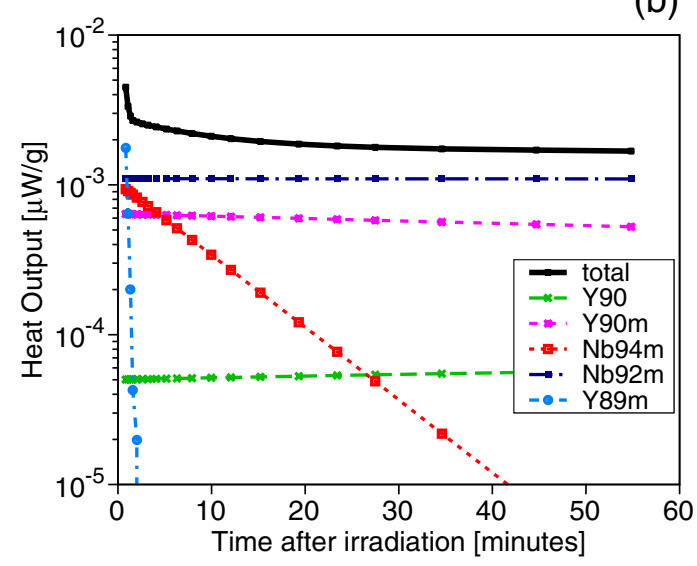

Figure 5. (a) Total decay-heat from niobium following a simulated 5-minute irradiation with two different nuclear libraries, and compared to the equivalent experimental measurement [12]. The grey-band surrounding the TENDL-2015 curve represents the simulation uncertainty, while the experimental error is given by the bars surrounding the data points. (b) the radionuclide-separated decay-heat for the TENDL-2015 simulation.

assessments. The ability to automatically generate plots to represent this complex information, based on the predicted time-evolution of the nuclide inventory from simulations, is a powerful asset - providing immediate and significant insight into the relative importance of different radionuclides as a function of time. Such plots also add to understanding when included as part of wider validation exercises and scoping studies, allowing the maximum amount of information from complex inventory simulations to be disseminated.

This work was funded by the RCUK Energy Programme [grant number EP/I501045]. To obtain further information on the data and models underlying this paper please contact PublicationsManager@ccfe.ac.uk

\section{References}

[1] J.Ch. Sublet, J.W. Eastwood, J.G. Morgan, M. leming, M.R. Gilbert, Tech. Rep. CCFE-R(11) 11 Issue 8, CCFE (2016), http: / / fispact . ukaea . uk

[2] R.A. Forrest, Fus. Eng. Des. 43, 209 (1998), http://dx.doi.org/10.1016/S0920-3796(98) 00418-9

[3] M.R. Gilbert, L.W. Packer, J.Ch. Sublet, R.A. Forrest, Nucl. Sci. Eng. 177, 291 (2014), http://dx.doi.org/10.13182/NSE13-76

[4] M.R. Gilbert, J. Marian, J.-Ch. Sublet, J. Nucl. Mater 467, 121 (2015), http://dx.doi.org/ 10.1016/j.jnucmat.2015.09.023

[5] T. Williams, C. Kelley, et al. (2015), current version of GNUPLOT $(5.0,2015)$ available from http://www . gnuplot.info/

[6] A.J. Koning, D. Rochman, J. Kopecky, J.Ch. Sublet, M. Fleming, E. Bauge, S. Hilaire, P. Romain, B. Morillon, H. Duarte et al., TENDL-2015, release Date: January 18, 2016. Available from https://tendl.web.psi.ch/tendl_2015/tendl 2015.html

[7] M.R. Gilbert, J.Ch. Sublet, A. Turner, Tech. Rep. CCFE-R(16)37, CCFE (2016), available from http://fispact.ukaea.uk

[8] U. Fischer, P. Pereslavtsev, A. Möslang, M. Rieth, J. Nucl. Mater. 386-388, 789 (2009), http://dx.doi.org/10.1016/j.jnucmat. 2008. 12.221

[9] R. van der Schaaf, F. Tavassoli, C. Fazio, E. Rigal, E. Diegele, R. Lindau, G. LeMarois, Fus. Eng. Des. 69, 197 (2003), http://dx.doi. org/10.1016/S0920-3796 (03) 00337-5

[10] R. Lindau, A. Möslang, M. Rieth, M. Klimiankou, E. Materna-Morris, A. Alamo, F. Tavassoli, C. Cayron, A.M. Lancha, P. Fernandez et al., Fus. Eng. Des. 75-79, 989 (2005), http://dx.doi. org/10.1016/j.fusengdes. 2005.06.186

[11] V. Barabash, Tech. Rep. HTN8X3, ITER organisation (2016)

[12] F. Maekawa, M. Wada, Y. Ikeda, Tech. Rep. JAERI 99-055. see also JAERI-Data/Code 98021 and JAERI-Data/Code 98-024, JAEA (1999), http://www.jaea.go.jp/jaeri/

[13] J.Ch. Sublet, M.R. Gilbert, Tech. Rep. CCFE$\mathrm{R}(15)$ 25, CCFE (2015), available from http://fispact.ukaea.ukx

[14] M.B. Chadwick, M. Herman, P. Obložinsky, M.E. Dunn, Y. Danon, A.C. Kahler, D.L. Smith, B. Pritychenko, G. Arbanas, R. Arcilla et al., Nuclear Data Sheets 112, 2887 (2011), https://doi.org/10.1016/j.nds.2011.11.002, http://www.nndc.bnl.gov/endf/b7.1/

[15] M.R. Gilbert, J.Ch. Sublet, Tech. Rep. CCFER(16)36, CCFE (2016), available from http://fispact.ukaea.uk along with other versions 